\title{
mHEALTH FROM THE PERSPECTIVES OF INDIAN RURAL POPULACE; URGENT NEED TO MAINSTREAM MHEALTH WITH THE EMERGENCE OF COVID-19
}

\author{
Ms. Manjula Venkataraghavan \\ Assistant Professor, Manipal Institute of Communication, \\ Manipal Academy of Higher Education, Manipal-576104. \\ Dr. Padma Rani \\ Professor\& Director, School of Communication, \\ Manipal Academy of Higher Education, Manipal-576104. \\ Dr. Lena Ashok \\ Associate Professor, MSW program, Prasanna School of Public Health, \\ Manipal Academy of Higher Education, Manipal-576104. \\ Dr. Padmakumar K \\ Associate Professor, Manipal Institute of Communication, \\ Manipal Academy of Higher Education, Manipal-576104 \\ Corresponding Author Email: padmakumar.k@manipal.edu
}

\begin{abstract}
mHealth now, more than ever, need to be integrated with existing health systems to further contactless healthcare and health delivery solutions. Studies have reported that mHealth can ensure enhanced health outcomes, ample patient care and offer quality support for professional health workers. Several studies have examined the potential of mHealth from various stakeholder perspectives. However, a serious dearth of studies exists that examine the advantages and challenges of using this technology from mHealth users' perspective especially in the rural context. This study examines the use of mobile phones for health-related purposes from the perspective of rural people belonging to the South Indian state of Karnataka. The focus is on their use of mobile phone to seek medical assistance, health information and services. The study also seeks to understand the barriers and challenges faced by the rural folk in accessing health related services, information and assistance through mobile phones. A qualitative research approach using in-depth interviews as data collection tool, was applied. Mobile phones are reported as useful by the rural mobile phone users for receiving health-related messages as well as for accessing help during emergency health conditions. However, to enable equity of use of this technology in the rural scenario and also for government to use this technology for providing responses during public health emergencies such as COVID-19, challenges such as the existing mobile phone infrastructure, digital illiteracy and perceived health concerns of mobile phone use among rural folk need to be addressed.
\end{abstract}

Key words: COVID-19, mHealth, mobile health, mobile phones, rural, healthcare

\section{INTRODUCTION}

The second wave of COVID -19 in India witnessed complete crumble of health infrastructures across the country. The number of deaths shot up to $1,85,000$, with the total number of infected cases reaching over 15.9 million by April 2021. (Jain, Iyengar \& Vaishya., 2021). Many state governments are planning to strengthen health infrastructures with new health facilities and construction of new oxygen plants ( Bhuyan, A., 2021). From the time World Health Organization (WHO) declared the novel corona virus outbreak as a global pandemic, health systems worldwide have been grappling with the challenge of providing contactless care and health delivery services (Jordan, 2020). Amidst this pandemic, mHealth or mobile health seem to have emerged as the way forward for health systems, enabling response and health delivery solutions without the fear of crosscontamination during outbreaks and other emergency health situations (Ye., 2020).

Several mHealth initiatives in response to the pandemic was launched in India too, to help 
increase public awareness of the virus and to enact measures to reduce its spread. However, a sound amalgamation of mobile phone use and smartphone applications with health service delivery, public health systems and healthcare administrations could have achieved a better management of the spread of the virus and better pandemic management (Pai, \& Alathur.,2020).

The use of mobile technology such as mobile phones, patient monitoring devices, personal digital assistants (PDAs), and other wireless devices in the field of medical and public health practices is called mHealth or Connected Health (WHO,2011). Evidence exists that mHealth can help in dispersing health information to rural and remote areas (Sharma, Shinde, \&Kar, 2017), serve reminders, provide an appropriate response to an emergency, monitor and bestow support (Sondaal et al.,2016). mHealth can help the user consult and seek an appointment with health professionals, access both test result as well as personal health information (Hoque,2016).

In India, large scale variations in health literacy, healthcare inequity and poor risk perceptions exists (Kar, Ransing, Arafat, \& Menon.,2021). Hence effective integration of mHealth solutions into existing public health systems would require deeper examination of the socio-economic aspects as well as existing infrastructural conditions. Further, successful implementation of mHealth tools would require evaluation of end user acceptability. Designing mHealth interventions to ensure successful outcomes would require among other things, analysis of perceptions of mHealth tools and its use among various stakeholders involved in the health sector. While the potential for the implementation of the technology has been examined from the health care and health care professionals' perspective, there is a dearth of studies that have examined the perceptions of mHealth from the users' perspective. Such studies would bring forth various unique aspects into the open which can then be addressed to design successful health tools, interventions and programs. This paper is based on an exploratory study conducted among rural mobile phone users belonging to the villages around the Udupi Taluk, Karnataka, India. In south Asia 'Taluk' is a term used for an administrative district, comprising of a number of villages. The research focuses on the rural mobile phone users' perspective of mHealth, the perceived advantages and barriers of mHealth technology especially the use of mobile phone to acquire medical help and health services by the rural populace.

\section{METHODS}

\section{Context}

Udupi district boasts of a good healthcare and human development indices. It also has a strong system of surveillance, monitoring and reporting (Umakanth, Bhat, Jagadeesha \& Shetty, 2021). Despite this , the district was one among the 17 districts of Karnataka that witnessed a steep increase in Corona virus cases in May 2021(India Today, May 16, 2021).

\section{Selection of sample}

Though data saturation is likely to occur within the first twelve interviews (Guest et al), a sample size of sixteen participants was taken from villages belonging to the Udupi Taluk using convenience sampling. This was done to balance the number of men and women participants in the sample.

The eligibility criteria for choosing the villages from where the participants were selected, was that the village had to be at a minimum of $10 \mathrm{Km}$ distance from the nearest multispecialty medical facility. The participants were selected based on their access to mobile phones as well as their ability to communicate in either English, Hindi or Kannada. Participants not fluent in English were interviewed in Kannada, the local language of Karnataka, with the help of a translator. All the participants had verbally consented to the interview and to the recording of the same.

\section{Data Collection}

An interview guide was used to understand the perceptions of mHealth among the participants for period of two weeks. The primary areas covered through the interviews are

i. Present healthcare management and the challenges faced by the rural populace with the health system.

ii. Current use of mobile phones.

iii. Current use of mobile phone for health purposes 
iv. Perceptions of benefits in the use of mobile phones for healthcare management

v. Barriers and challenges in the use of mobile phones.

A review of mHealth literature enabled in curating questions to explore and elicit information in the above areas. Field notes of the in-depth interviews were made. All the conversations were between 30 to 45 minutes duration and were conducted in the residences of the participants or at their places of work.

\section{ANALYSIS}

The data analysis was conducted using grounded theory methodology. Grounded approach is usually followed to understand social processes and allows for theory formation based on the data which is collected and analysed (Noble \& Mitchell., 2016). A thematic analysis was conducted alongside data collection to help constant comparison of data. New themes that arose from the raw data were then compared with previous interview data to identify and develop key inductive themes. No new codes were added after the tenth interview as data saturation was attained by then.

\section{RESULTS}

Healthcare management and the challenges faced within the health system.

Preference of private clinics/health facility over government health centres

Almost all the participants interviewed preferred going to private institutions for their health requirements. Nine of the participants prefer going to a clinic near their place of stay, and six of them prefer to go to a significant multi-specialty private health facility near their area. Only one participant from the study said that he and family visit the PHC in his village whenever required.

The reasons given by the participants for preferring private medical facility over government medical facilities (PHC/UHC) ranged from the shortage of doctors, coverage under the medical scheme offered by the employer as well long waiting time. About half the participants of this study were employed and were covered by the Employees' State Insurance scheme (ESI) as well as the health schemes offered by their employer. One participant informed that during her pregnancy though she was registered at the local $\mathrm{PHC}$ and received regular SMS reminders for intake of iron\& folic acid tablets and for periodic antenatal check-ups etc. from the health workers. Though she still chose to deliver her baby at a private facility due to overcrowded PHC as well as the non-availability of doctors most of the time. Many participants expressed that the doctors are either not allocated by the government, or have too many patients waiting for consultations or are just not available for them as they are busy attending to other medical emergencies.

"Whenever my children, husband or I have a fever or any other medical problem, I visit this clinic which is more convenient than visiting the PHC where the doctor might not be available or if available might have too many patients to attend "(Participant number 8, Feature phone user)

The only participant of the study who visits the local PHC for his medical requirements, opined that the situation has improved in the PHC of his area lately. According to him, this could have happened due to increased governmental focus on improving the situation.

Use of mobile

Preference of basic feature phones over smart phones

Most of the nine participants belonging to the age group $40-60$ years used basic mobile feature phones. Only one participant belonging to this age group was a graduate while the rest had just completed various levels at school. All the seven participants belonging to the age group 20-40 years used smartphones. Among these seven participants, four were women. Two out of the seven smartphone users were post-graduates, while three among them were graduates. Out of the remaining two smartphone users, one had a diploma in teaching, while the other had just completed school.

\section{Dominant use of making \& receiving calls}

Participants using feature phones reported their use of mobile mainly for the primary purpose of making or receiving calls, only two of them said they use their feature phones for sending and receiving SMS messages as well. "...my mobile phone is only for making \& receiving calls..." (Participant number 10, Feature phone user) 
Most participants who used feature phones prefer only voice calls over SMS for any purpose. The reasons for this preference was found to be mostly due to technical illiteracy, lack of interest to use the device for texting purpose as well as a perceived lack of time for texting or reading text messages. However, some participants felt that text messages are advantageous over calls as text messages can be read at their convenience and can be reread for clarifications and can also be forwarded to others when required.

\section{Use of internet or internet based apps by smart phone users.}

None of the feature phone using users reported use of internet or internet based apps. While most participants using smartphones reported using their device to access the internet and also informed that they use webbased applications such as WhatsApp, Facebook, and shopping apps such as flip kart along with other regular purposes of the mobile phone such as contacts, calendar etc. The internet is reported to be used for downloading work-related files and documents, for accessing news and listening to music, seeking health information, for uploading documents to government sites (Income tax, passport office), Bank, Taluk office, etc.

Many participants felt that mobile phones were very useful in accessing relevant health information as well as for receiving essential messages from the government such as taxrelated reminders, submission of pertinent documents to authorities, communication from their children's schools, as well as for booking cooking gas cylinders etc. Most participants informed that mobile phones help them to keep in touch with family, relatives and friends and one participant said that it also helps in reporting ahead when she gets late for work. Almost all the participants felt that mobile phone had become an essential part of their lives.

“On my mobile phone, I get information about what is available at the ration store for a particular month, I also get messages from the cable operator. I send and receive SMS from my children, this is important because, I am aware of their whereabouts. Today mobile phone for me has become important, without mobile phone everything comes to a standstill" (Participant number 8, Feature phone user)

Current use of mobile phones in healthcare and management

Use of mobile phone to contact health system

All the participants of the study expressed that mobile phones helped them contact medical help in times of emergency, to call doctors for medication or first aid, for ambulance, cabs, auto as well as their neighbours, friends and family for assistance to transport the sick or injured.

"When my father became suddenly ill, I was able to summon an ambulance and medical help by dialling 108 through my mobile phone."(Participant number 6, Smart phone user)

Some participants opined that mobile phones are useful in calling medical facilities ahead to check the availability of doctors before travelling to the clinic or in fixing appointments for consultations.

"My 83-year-old mother also uses her mobile phone to call the doctor to fix appointment beforehand."(Participant number 7, Feature phone user).

\section{Need for physical consultation}

While some participants call their physicians during an emergency (sick child/adult, fracture etc.) to confirm medicine and dosage with their regular doctors, many believed in directly going in for consultations whether the requirement is urgent or otherwise, as they felt that consultation with doctors always had to be in person. The reason expressed for this was that through mobile phones they may not be able to convey their health issues accurately to the physician, and also receive the health advice given to them in its entirety.

Two participants from the study spoke about the SMS based health information, and reminders they received from the local PHCs during their pregnancy as part of the government initiated Mother and Child Tracking System (MCTS) program. The reminders were directed at expectant mothers reminding them to take iron and folic acid tablets, for regular intake of nutritious food, for tetanus injection and periodic antenatal 
check-ups. Also to young mothers for the need for timely immunization of babies. This service according to them was very useful and enabled in taking proper care of themselves during pregnancy and also regular vaccination of their babies.

Use of the Internet to access health information.

Smartphone users among the participants reported using the internet regularly to access and clarify health-related information such as side effects of medicines prescribed, banned medicines, symptoms of diseases and precautionary measures. One woman participant who was a smartphone user, spoke about how she used the internet to clear her concerns about her nutritional requirements, supplements during her pregnancy and later to clear concerns over the vaccination requirements for her child.

"I used to browse online while I was pregnant for calcium substitute, for the required haemoglobin count that I needed to maintain as well the required weight gain during each trimester. After the birth of my baby, I had a query regarding the BCG vaccination. On checking it was found that BCG which was to be given at birth was not administered to my baby. So I checked with the doctor too about the same...I also checked about optional vaccines of Chicken Pox and Rotavirus to understand if I have to have it administered to my child certainly".(Participant number 6, smart phone user)

One participant reported using WhatsApp for sending messages from blood donation camps to his regular donor groups on his contact list.

\section{Benefits anticipated through mHealth}

Improve accessibility to health information

All the participants of the study agreed that mobile phones have become a necessity of life and that almost everyone has access to this technology. Participants unanimously believed that mobile phones could enable better access to health-related information. When asked about the type of health information preferred, few participants opined that data from government health departments relating to the health schemes, programs and policies will be useful in creating more awareness of the facilities and provisions provided by the government. Such information can then be spread among those who do not have access to it. Participants also suggested that SMS information on communicable diseases such as dengue, malaria, HIV AIDS etc., the precautionary measures to be taken to avoid contracting the prevalent diseases as well as details on noncommunicable diseases, healthy eating habits and nutritious diets during ailments will also be useful.

Suggestions from participants included periodic mobile based video messages from health departments on first aids in case of fracture, stroke, and care for patients undergoing physical/mental trauma etc. may enable better and informed caregiving by family members. Many participants preferred such messages in the form of either text messages or as pictorial or audio-video messages. Several participants using feature phones preferred SMSs in their local language, Kannada.

"Dietary information from medical centres for specific ailments through SMS will aid in recouping and taking proper care especially for diabetes, hypertension and cholesterolrelated ailments." (Participant number 16, Smart phone user)

It was felt that regular SMS updates on local health centres with names of physicians and their phone numbers for specific medical emergencies by the government health department could be useful too. Information on banned medicines should regularly be sent by the government through SMS, as this can be read and forwarded to others and can also inform others who do not have access to the information.

“... then we will know about banned medicines, why they were banned and why certain medicines are unavailable ..." (Participant number16, Smart phone user)

\section{Provide Reminders}

Some participants reported that the community health workers or nurses from the local PHC call them to inform them about upcoming government health programs. Two participants spoke about the reminders they received during their pregnancy period from the local PHC as part of the MCTS program, reminding them to take iron \& folic acid 
tablets, nutritious food as well as to tell them of an upcoming check-up. Hardly any reminders through mobile related to health management were reported by the other participants.

"If a reminder comes one week before the mandatory health check-up (for the wife, a heart patient) it will be beneficial, as both of us are working, it will help us schedule and plan for a check -up date much ahead...."(Participant number 16, Smart phone user)

Barriers and challenges to the use of mobile phones for healthcare management

Digital illiteracy

Among the sixteen participants of the study, majority were feature phone users, compared to the smartphone users. Participants using feature phones were only using their device for making or receiving calls and prefer just voice calls over SMS for any purpose. Only two of them reported the use of their feature phones for sending and receiving SMS messages. Some of the feature phone users exhibited digital illiteracy. They did not know to use their phone for any other purpose other than making or receiving calls.

\section{Health fears}

Some participants expressed concern over the effects of mobile phone use on their health. One participant who had a heart surgery feels that keeping mobile in his breast pocket could harm his health from the radiation that is emitted. Some participants were also concerned about the impact of excessive use on one's eyesight. Few were worried about the effects of overuse of mobile phones by the young users.

"Mobile phones are problematic as it results in constant chatting, unnecessary messaging, sharing of pictures etc. among younger children which could lead to blackmailing, bullying etc....." (Participant number 3, Feature phone user)

Such predisposition and concern are likely to become a barrier to their use of mobile phones in healthcare.

\section{Need for physical consultation}

Many participants felt that physical consultation with a doctor cannot be replaced. Physical meetings help in clearly telling the doctor about one's health issue as well as in taking the health advice completely.

"In-depth conversation about the health problem is possible when I have an ailment say a cough, in detail explanation is possible in person...." (Participant number 14, Feature phone user)

\section{Infrastructure challenges}

Many participants spoke about the connectivity issues faced by them in their mobile phone use.

"Only mobile networks of BSNL and Airtel seem to work here. The calls get connected much better than the broadband connections, which is very slow here" (Participant number 5, Smart phone user)

Power cut was another big issue faced by the participants especially during rainy seasons when there is no electricity for two to three days in a row. Charging their handsets during such times becomes a challenge.

Nearly half the number of participants spoke about lack of public transport into the interiors of their village, which makes it especially difficult when someone falls sick at night or during adverse weather conditions.

"The place used to be like an island until about four years ago, no proper transport, communication facilities etc. Today roads are better, but transport facility at nights is still a problem. The coming of mobile phones has brought some relief to this situation" (Participant number 7, Feature phone user)

All participants unanimously agreed that emergencies are times when mobile phones come in handy. During medical emergencies, mobile phones help them connect with doctors or nurses to seek some remedy; to contact auto drivers; ambulance services; neighbours, friends with vehicles to help in transporting the sick person to the nearest medical help during an emergency. Some participants preferred rushing to a medical facility directly. Few informed that they stock up medicines for primary and other ailments on a regular basis. As the transport during nights as well as during rains become difficult, they call up nurses or doctors when situations arise, for 
advice on dosage of the medicines to be consumed.

"During night times when my children or any family member develop fever, cold or upset stomach, I call my doctor belonging to the clinic where I have been visiting since childhood and check the dosage of medicine to be given and administer accordingly" (Participant number 7, Feature phone user).

\section{DISCUSSION}

Under the current pandemic situation of COVID-19, mHealth has turned into a vital service(Neubeck et al., 2020). All the participants agreed that mobile phones can be put to use by the government to disperse crucial health-related information and messages. This fact translates to the high level of acceptance of mobile phones as a possible tool for health-related information as well as a tool that would enable them to some extent in self-management of health. This finding supports the findings of Smith et al., 2015. This finding also reinforces the fact that mobile phone is a plausible tool for sending health information as well as a device that can support health interventions, especially in the rural, remote communities (Ndayizigamiye \& Maharaj, 2017; Feinberg et al., 2017). Further, use of mHealth during pandemics help solve problems related to social isolation and decreased physical activity (Neubeck et al., 2020).

An important finding of the study is that large number of rural mobile phone users were predominantly feature phone users, educated only up to high school and were using it for only making or receiving calls. Most of them were not even able to use all features of the phone including the use of texting services. So if mHealth use and applications have to improve, then steps to educate people about the use of all the features of the phone have to be initiated either by the government, service providers or health centres, both public and private. Access to the internet through mobile phones was found to be very insignificant, and there is hardly any use of internet-based apps. Most of them are not interested in accessing the internet and are content in using their phones for only the primary purpose of making and receiving calls.
They were satisfied with their devices and showed no interest in switching to smartphones. This aspect can become a barrier in maximizing the use of this technology to access health information and services. While all the participants of the study using smartphones were graduates under the age of 40 years and were very comfortable using their smartphones to go about their daily information and communication requirements. This finding reinforces earlier mHealth findings that people under 40 years used technology with more ease and consistency (Boodoo et al., 2017). This inconsistency in technology use among the populace will pose a challenge in fulfilling needs of the target users and cannot be ignored while designing mhealth interventions (Alvarado, et al., 2017; Boodoo et al., 2017). Several other studies have identified such lack of acquaintance with technology as a barrier to mHealth adoption (Fischer et al., 2014; Granger et al., 2016). However according to Smith et al., 2015, this concern would be valid only for a short period as the technically literate generation moves on in age to become the more substantial part of the population. Hence this technological barrier regarding usability may lose its pertinence over the years.

However, for successful implementation of mHealth programs and interventions, the targeted population must be carefully examined for the attitude and perception of this technology and its use by them as that would have a direct influence on its adoption by them. Studies to understand the user acceptance, needs attitudes and behavior toward mobile phone technology will help in augmenting the health intervention design (Smith, et al., 2015; Boodoo et al., 2017) .

Three significant uses emerged from this study in which mobile phone technology can offer respite in the existing scenario of overburdened healthcare and health delivery systems as well as infrastructural challenges. They are the use of mobile for a) Disseminating varieties of health-related information b) As reminder tools for taking medications and appointments and c) For providing emergency medical services over the mobile phone. 


\section{Disseminating varieties of health-related information}

All the participants of this study felt that mobile phones should be used by the government to disperse health information related to both communicable and noncommunicable diseases, its preventive measures and other health-related information such as nutritional requirements across ages. Participants also pointed out the need to spread awareness and knowledge about diverse health issues, pertaining to all age groups, so that it could be beneficial to all. Participants also pointed out the need to be informed about banned medicines. When such information can be disseminated through mobile phones, people would be wellinformed on banned drugs and avoid its use. Health messages especially information pertaining to first aid treatment in cases of grievous injuries, stroke, heart attack etc., in an audio-visual form also was suggested by some participants. Dissemination of information through mobile phones could lead to increased awareness of health-related aspects and issues which in turn can result in a healthier population with minor incidences and prevalence of preventable disease forms.

\section{As reminder tools for taking medications and appointments}

SMS reminders to take pills can be handy for maintaining continuity of drugs as well as to tackle forgetfulness which is a significant barrier to medical adherence (DeSouza et al., 2014). Studies have indicated the effectiveness of SMS reminders sent on mobile phones to keep up the adherence to medications and appointment attendance (Kannisto, Koivunen, \& Välimäki, 2014).

Participants of this study also considered reminder messages through mobile phones for taking medicines, for health check-ups and upcoming consultations as highly useful. Though at present a few ventures such as the SMS program under the Indian government's Mother and Child Tracking System (MCTS) program, Kilkari, T.B Missed call service and M-Cessation. All of these programs were launched in the Northern states of India; there is hardly any much such facilities in the south except for the SMS program under MCTS. Under the MCTS program, mobile phones are being used to keep track of young mothers and infants among the urban and rural populace. A study conducted in Pune, Maharashtra to check the use of MCTS text message service found that unless a local language which is simple and easy to understand is used for SMS messages the reception and use of the same can be very insignificant (Sharma A et al., 2016). Several participants of this study also preferred text messages to be in their local language. A few suggested the need for audio-visual messages that would explain specific health information that would be useful for administering first aid care during accidents, emergency health situations such as heart attacks, strokes etc.

\section{For providing emergency medical services over the mobile phone}

In times of emergency, when access to medical help becomes difficult, mobile phones help in getting the necessary help which otherwise might have been challenging especially in adverse weather conditions. The study also unveiled how the technology becomes useful in these remote rural areas in times of emergency to eliminate travel and yet receive medical guidance over the mobile phone. To call for an ambulance or other forms of transport to take the patient to the hospital, the technology has enabled some relief which was not available before the coming of this technology. Efforts can be directed by the government to have emergency medical health services such as call-in services to receive essential initial treatment solutions through mobile phones which people can seek and access at all times including emergency situations.

The usability aspect of the mobile phones shows a difference with the age group. While the older participants used basic handsets and preferred calls over SMS, participants of the younger age group were comfortable in accessing the internet through their smartphones. They were found to seek healthrelated information on a regular basis from online sources. This aspect is noteworthy and has to be kept in mind while devising mobile based solutions for the older technologically challenged population. Though this concern would be valid only for a short period as the technically literate generation moves on in age to become the more substantial part of the population. Thus technological barrier regarding usability may lose its pertinence over the years (Smith et al., 2015). 
Half the participants of this study were women and half out of these were employed. This device was found be useful unanimously by all the women who felt that mobile phones helped them to keep in touch with family and friends, helped them save time, energy as well as money and assist them in getting medical aid during times of emergency. These findings indicate that mobile phones not only has immense potential to supplement healthcare delivery and service to people living in remote rural areas but also can significantly empower women.

Concern about the adverse health effects such as radiation fears could act as a barrier to mHealth adoption. A review of the available epidemiological evidence indicates that mobile phone radiation could be carcinogenic (Hardell, 2017). Until the emergence of conclusive evidence on the effects of mobile phone radiation, this concern is likely to remain a barrier to its use.

Studies have reinforced the significance of face-to-face patient-physician contact in disease management (Chang et al.2013). The need for a physical check-up cannot be overlooked, as certain health conditions might require an analysis of signs and underlying context to enable a proper diagnosis. This study unravelled the extent of mobile phone use, types of health information sought by the people from this community as well as possible barriers to mHealth use. Successful awareness of health-related data can be achieved in this populace by factoring this information into mHealth intervention designs. Employing SMS based messages in the local language will also be highly received. Given the ubiquity of mobile phones in India, it makes it an available tool for supplementing the existing healthcare system (DeSouza et al, 2014) . However infrastructural challenges such as network coverage and power supply are still present as barriers to its full use.

\section{Areas for future research}

M-health shows potential to improve access and equity to health care delivery and services in remote rural areas by providing the muchneeded support mechanism to the community health workers (Strachan et al., 2012). However, a deeper insight into beneficiary perceptions regarding mHealth in specific health issue categories might be beneficial to understand the full potential of the medium to supplement the existing health system. Similarly, an insight into physicians' perception of benefits and barriers of mobile phone use for health purposes also need to be considered for designing better mHealth interventions.

\section{CONCLUSION}

The study found that information regarding disease awareness, preventive measures and nutrition are some of the requirements among rural mobile phone users. Benefits such as reminders for adherence to medication and appointments with doctors seem to be well accepted by the rural mobile phone users. While the older groups preferred voice calls over text messages, the need to factor this into mHealth interventions might be required till most of the population become comfortable using the technology for purposes other than receiving and making calls.

Health communication addressed to women may help them in taking informed healthrelated decisions. Such communications will serve as action calls for advancing their health. Further research in mHealth can be directed towards examining the barriers faced by mobile phone users in certain health ailment categories. Research studies may also be directed to probe the perception of mHealth obstacles from the physicians' perspective

\section{REFERENCES}

Alvarado, M. M., Kum, H. C., Coronado, K. G., Foster, M. J., Ortega, P., \& Lawley, M. A. (2017). Barriers to remote health interventions for type 2 diabetes: a systematic review and proposed classification scheme. Journal of medical Internet research, 19(2)

Bhuyan, A. (2021). Experts criticise India's complacency over COVID-19. The Lancet, 397(10285), 1611-1612.

Boodoo, C., Perry, J. A., Hunter, P. J., Duta, D. I., Newhook, S. C. P., Leung, G., \& Cross, K. (2017). Views of patients on using mHealth to monitor and prevent diabetic foot ulcers: qualitative study. JMIR diabetes, 2(2), e22.

DeSouza SI, Rashmi MR, Vasanthi AP, Joseph SM, Rodrigues R (2014) Mobile Phones: 
The Next Step towards Healthcare Delivery in Rural India? PLoS ONE 9(8): e104895. doi:10.1371/journal.pone.0104895

Feinberg, L., Menon, J., Smith, R., Rajeev, J. G., Kumar, R. K., \& Banerjee, A. (2017). Potential for mobile health (mHealth) prevention of cardiovascular diseases in Kerala: A population-based survey. Indian Heart Journal, 69(2), 182-199.

Fischer, S. H., David, D., Crotty, B. H., Dierks, M., \& Safran, C. (2014). Acceptance and use of health information technology by community-dwelling elders. International journal of medical informatics, 83(9), 624-635.

Granger, D., Vandelanotte, C., Duncan, M. J., Alley, S., Schoeppe, S., Short, C., \& Rebar, A. (2016). Is preference for mHealth intervention delivery platform associated with delivery platform familiarity? BMC public health, 16(1), 619.

Guest, G., Bunce, A., \& Johnson, L. (2006). How many interviews are enough? An experiment with data saturation and variability. Field methods, 18(1), 59-82.

Hoque, M. R. (2016). An empirical study of mHealth adoption in a developing country: the moderating effect of gender concern. BMC medical informatics and decision making, 16(1), 51.

Hardell, L. (2017). World Health Organization, radiofrequency radiation and health-a hard nut to crack. International journal of oncology, 51(2), 405-413.

Jain, V. K., Iyengar, K. P., \& Vaishya, R. (2021). Differences between First wave and Second wave of COVID-19 in India. Diabetes \& Metabolic Syndrome.

Kannisto, K. A., Koivunen, M. H., \& Välimäki, M. A. (2014). Use of mobile phone text message reminders in health care services: a narrative literature review. Journal of medical Internet research, 16(10), e222.

Kar, S. K., Ransing, R., Arafat, S. Y., \& Menon, V. (2021). Second wave of COVID-19 pandemic in India: Barriers to effective governmental EClinicalMedicine, 36.)
Neubeck, L., Hansen, T., Jaarsma, T., Klompstra, L., \& Gallagher, R. (2020). Delivering healthcare remotely to cardiovascular patients during COVID19: A rapid review of the evidence. European Journal of Cardiovascular Nursing, 1474515120924530.

Ndayizigamiye, P., \& Maharaj, M. (2017). Determinants of Mobile Health Adoption in. The African Journal of Information Systems.

Noble, H., \& Mitchell, G. (2016). What is grounded theory?. Evidence-based nursing, 19(2), 34-35.

Pai, R. R., \& Alathur, S. (2020). Mobile health intervention and COVID-19 pandemic outbreak: insights from Indian context. International Journal of Health Governance

Rao, K., \& Ramani, S. (n.d.). HUMAN RESOURCES FOR HEALTH IN INDIA: CURRENT CHALLENGES ... Retrieved November 11, 2016, from https:/ / www.idfc.com/pdf/report/2013 -14/Chapter-20.pdf

Smith, R., Menon, J., Rajeev, J. G., Feinberg, L., Kumar, R. K., \& Banerjee, A. (2015). Potential for the use of mHealth in the management of cardiovascular disease in Kerala: a qualitative study. BMJ open, 5(11), e009367.

Strachan.D.L. et al,2012. Interventions to Improve Motivation and Retention of Community Health Workers Delivering Integrated Community Case Management (iCCM): Stakeholder Perceptions and Priorities.A m. J. Trop. Med. Hyg., 87(Suppl 5), 2012, pp. 111-119 doi:10.4269/ajtmh.2012.12-0030

Sharma, A., Shinde, A., \& Kar, A. (2017). Prevalence in the utilization of text message services under the mother and child tracking system of India: a cross sectional study from Pune district, Maharashtra, India. International Journal Of Community Medicine And Public Health, 3(8), 2319-2324.

Sondaal, S. F. V., Browne, J. L., AmoakohColeman, M., Borgstein, A., Miltenburg, A. S., Verwijs, M., \& Klipstein-Grobusch, K. (2016). Assessing the effect of mHealth interventions in improving maternal and 
neonatal care in low-and middle-income countries: a systematic review. PloS one, 11(5), e0154664.

Umakanth, S., Bhat, P., Jagadeesha, G., \& Shetty, A. (2021). Bracing for the pandemic: The udupi-manipal experience. Indian Journal of Respiratory Care, 10(4), 3.

WHO (World Health Organisation).2006. 'Working Together for Health-A World Health Report'. Geneva: World Health Organisation.

World Health Organization. (2014). mHealth: New horizons for health through mobile technologies: second global survey on eHealth. Geneva, Switzerland: World Health Organization, 2011.

Ye, J. (2020). The role of health technology and informatics in a global public health emergency: practices and implications from the COVID-19 pandemic. JMIR Medical Informatics, 8(7), e19866.

http://www.pib.nic.in/newsite/PrintRelease. aspx?relid=134503

www.livemint.com > Industry > Telecom

https://www.indiatoday.in/india/story/17districts-in-karnataka-seeing-covid-19cases-rise-pm-modi-to-chair-virtualmeet-with-dms-1803083-2021-05-16

https://www.google.com/search?q=covid + ca ses+in+india\&rlz=1C1CHBD_enIN905IN 905\&oq $=$ covid\&aqs $=$ chrome. $2.0 j 35 i 3912 j 01$ $5.5304 \mathrm{j} 0 \mathrm{j} 8 \&$ sourceid $=$ chrome $\& \mathrm{ie}=\mathrm{UTF}-8$

https:/ / timesofindia.indiatimes.com/city/ma ngaluru/covid-19-cases-continue-tosurge-in-dkudupi/articleshow/77043021.cms

https://www.bloombergquint.com/business/ 2017/05/22/indias-mobile-phonesubscriber-base-inches-close-to-a-billion 